\title{
Fluoridated elastomers do not reduce plaque around orthodontic brackets
}

\author{
What is the effect of fluoridated elastomers on the quantity of disclosed dental \\ plaque surrounding an orthodontic bracket?
}

\begin{abstract}
Benson PE, Shah AA, Campbell IF. Fluoridated elastomers: effect on disclosed plaque. J Orthod 2004; 31:41-46

Design This was a randomised, prospective, longitudinal clinical trial employing a split-mouth, crossover design.

Intervention Fluoridated elastomers were randomly assigned at the first visit to be placed around brackets on the upper left lateral incisor, upper left central incisor and lower right canine, or the upper right lateral incisor, upper right central incisor and lower left canine. Nonfluoridated elastomers were placed on the contra-lateral teeth. After 6 weeks (visit 2) the elastomers were removed, the teeth disclosed and a photograph taken. Non-fluoridated elastomers were placed on all brackets for one visit to allow for a washout period of at least 6 weeks. At visit 3, fluoridated elastomers were placed on the contra-lateral teeth to visit 1 . After six weeks (visit 4), the procedures at visit 2 were repeated.
\end{abstract}

Outcome measure Area and proportion of the buccal surface covered with disclosed plaque was measured using computerised image analysis.

Results A total of 333 images were collected from 34 patients. The only significant independent variable for the area of disclosed plaque was the subject $(P<0.001)$. The significant independent variables for the proportion of disclosed plaque were the subject $(P<0.001)$ and the tooth type $(P=0.002)$. The independent variable describing the use of fluoridated or non-fluoridated elastomers was not significant for either the area or the proportion of disclosed plaque.

Conclusions Fluoridated elastomers do not affect the quantity of disclosed plaque around an orthodontic bracket.

\section{Commentary}

This is an elegant study focusing on the important issue of plaque build-up and enamel demineralisation around orthodontic brackets in fixed-appliance therapy. Local release of fluorides in an orthodontic environment has already been studied extensively and some clear definitions have emerged. Teeth with fluoridated elastomers have a harder enamel surface, which is presumably more resistant to enamel demineralisation, as shown in previous work. ${ }^{1}$ Wilson and Gregory ${ }^{2}$ have also established that there is a reduction in severity and incidence of enamel demineralisation and an initial reduction in Streptococcus mutans count. The authors here have a clear purpose in evaluating the beneficial effects in a clinical environment for a relevant period of time.

The study was structured on well-defined criteria and the randomisation is adequate. The enlargement of the sample size and the fact that all the participants in the trial were accounted for speaks of a critical balance in the trial. As the authors candidly highlighted, the crossover design in such a trial may prove to be its weak point, especially since the slow release of fluorides from elastomers may be absorbed on the control teeth and thereby bias the results. The issue of a smaller sample size was also critically discussed and justified. The only area of concern therefore is in the visualisation of images and digital subtraction of the bracket image prior to measurement. The dexterity of using a mouse to draw around a bracket accurately is an issue. This is not easy at the best of times and the increase in random error compared with previous studies highlights this.

As with any research, however, questions remain. Is the measurement of disclosed plaque around an orthodontic bracket adequate to assess the quantity of plaque build up? Can plaque build up be quantitatively measured only in the surface area or is the depth of the plaque film relevant? Would the adjunctive estimation of Streptococcus mutans counts have been more accurate in monitoring the effects of the fluoride-release phenomenon?

In addition, what is the obvious clinical implication of the present study? If the use of fluoridated elastomers does not affect the quantity of disclosed plaque should one recommend their use to clinicians on the basis of the evidence available? Information is not the same as knowledge. To extract one from the other, as the word suggests, one must inform. The knowledge must be transmitted without uncertainty or confusion. Even if the build-up of plaque was not reduced, it has been shown that the use of fluoridated elastomers would benefit the patient in terms of controlling enamel demineralisation. ${ }^{3}$

\section{Practice point}

- Fluoridated elastomers do not affect the quantity of disclosed plaque but may help control enamel demineralisation.

\section{Anmol Kalha}

Department of Orthodontics and Dentofacial Orthopaedics, College of Dental Sciences, Davangere, Karnataka, India

1. Banks PA, Chadwick SM, Asher-McDade C, Wright JL. Fluoride-releasing elastomerics - a prospective controlled clinical trial. Eur J Orthod 2000; 22:401-407.

2. Wilson TG, Gregory RL. Clinical effectiveness of fluoride-releasing elastomers. I: salivary Streptococcus mutans numbers. Am J Orthod Dentofacial Orthop 1995; 107:293-297.

3. Benson PE, Parkin N, Millett DT, Dyer FE, Vine S, Shah A. Fluorides for the prevention of white spots on teeth during fixed brace treatment. Cochrane Database Systematic Rev 2004;(3).

Evidence-Based Dentistry (2004) 5, 96.

doi:10.1038/sj.ebd.6400299 